\title{
Personalized customization system solution using augmented reality technology
}

\author{
Liying Feng ${ }^{1,3}$, Liyao $\mathrm{Ma}^{2}$ and Giapweng $\mathrm{Ng}^{3, *}$ \\ ${ }^{1}$ Hainan Vocational University of Science and Technology, Hainan, China \\ ${ }^{2}$ Faculty of Art and Design, University of Hainan, Hainan, China \\ ${ }^{3}$ Faculty of Computing and Informatics, University Malaysia Sabah, Malaysia
}

Keywords: Augmented reality, Kinect somatosensory technology, Virtual fitting, Interactivity, Experiencing, Clothing customization.

\begin{abstract}
This paper presents a personalized clothing customization system using Augmented Reality (AR) technology. The goal of this paper is creating a more interactive and immersive experience for tailor designer by bridging physical personalized clothing customization and digital resources. This system can make the traditional clothing customization process more convenient and efficient and has strong interaction and experience. At the same time, it reduces the time cost, labor cost and material cost of sample making in the traditional clothing customization process. This paper discusses the feasibility of the system, introduces the system development, testing, and application environment, the overall system design scheme and system workflow, and details the specific functions of each module. It also introduces the system detection methods and observation indicators. The system uses technologies such as augmented reality (AR) technology, Azure Kinect somatosensory technology, and OpenGL 3D rendering, combined with the functional advantages of the somatosensory virtual fitting system, focusing on the improvement and role of AR technology in the clothing customization process, effectively improving user satisfaction with clothing customization and saves production time and costs.
\end{abstract}

\section{Introduction}

In the process of actual costume customization, how to make customers participate in the design process effectively is the difficulty of customization. Sufficient and effective communication between users and designers is the premise to guarantee the satisfaction of design works, is the most critical link in the design process, and is also the most difficult to achieve. At the same time, in the process of practical costume customization, how to quickly make the user try on and experience the sample effect of customized clothing is also a

\footnotetext{
"Corresponding author: nggiapweng@ums.edu.my
} 
problem in customization, because the sample production not only takes time, but also increases the cost of labor and materials, and if the user is not satisfied, the improvement of production needs to continue to increase the time and cost of production. For this reason, how to enable users to participate in the design process, experience design effects, and save production time and costs is a problem faced by modern clothing customization, and it is also the basis and trend to achieve future mass customization. [1]

\section{Feasibility analysis}

\subsection{Increasingly mature technical support}

Augmented reality (AR) technology is to superimpose electronic elements such as virtual images, animations, and audio in real scenes, giving a richer and more authentic experience. AR technology plays a very important role in product design, display, tour guide, training and maintenance, remote collaboration, game experience, etc. with its powerful experience, rich interaction, and various display functions. AR technology provides interactive scenarios for people's production activities, reduces input costs, and simulates high-risk environments, effectively avoiding dangers. At present, it has gradually integrated into the application stage of people's life and production process from the laboratory research stage.

Using AR technology, 2D clothing design drawings can be converted into 3D clothing models for users to directly view the design styles. Using AR technology, users can realize the independent coloring and color matching of clothing colors and can directly observe the effect of colored clothing. Using the rendering function of AR technology can make the digital cloth in the computer more realistic. At the same time, with auxiliary equipment such as motion capture and tracking, AR technology can also overlay digital clothing in real-time scenarios (that is, on the customer or the model's body), and even the clothing can follow the different actions of the customer to show the corresponding status, and finally to achieve the effect of real-time interaction with users, try-on experience.

At present, the advent of the $5 \mathrm{G}$ era provides a wider platform and guarantee for the application of AR technology, effectively reduces the delay of AR display effects, and also improves the picture quality of cloth simulation and rendering effects. It can provide users with faster and better clothing visual effects. In terms of hardware, sensors (such as Azure Kinect) used for image recognition and motion tracking are more mature and advanced, providing people with high-quality capture effects. It is particularly worth mentioning the Unity 3D platform. According to Michelle, M. (2012), the Unity 3D platform is a comprehensive game development tool. It provides users with the ability to create interactive content, such as 3D games, visual architecture, 3D real-time animation, etc [2]. It is a fully integrated professional game engine. we can take advantage of its capabilities to simplify the development process. The development of these technologies provides strong technical support for the development of the AR clothing customization system.

\subsection{Broad market demand}

With the progress of science and technology and the development of The Times, the development speed of e-commerce in the role of the Internet is amazing. In 2018, according to PAR Byron Consulting (2018), the online transaction volume of "double 11" in China has exceeded RMB 314.3 billion Yuan [3]. The top three industries of online retail sales are clothing and apparel, household appliances and home furnishing, among which the retail sales of clothing and apparel account for $20.3 \%$, far ahead of other industries. From the 
perspective of consumption, these data intuitively show the huge potential of the consumption market and extraordinary creativity. This is enough to show the influence of clothing on people's lives.

On the other hand, through the comprehensive analysis of consumption information by experts, the trend of high-end, personalized and diversified upgrading of consumer demand is also fully reflected. If today's high volume of transactions is enough to prove that e-commerce has reached the red sea era, then high-quality, high-end, personalized, diverse and experience-based interactive services will be the key to the next blue ocean era. In 2019, according to China Business Industry Research Institute (2019), the online sales volume of "double 11" in China has exceeded RMB 410.1 billion Yuan, and the top three retail sales sectors are personal care, beauty makeup, and snack food [4]. Clothing and apparel products are outside the top 8 in the retail industry, which is enough to prove that people's online clothing purchase has reached a peak and gradually shifted to more elegant and unique personalized customized clothing, according to the forecast of apparel turnover in 2018, it will be a new market of more than RMB 60 billion Yuan.

Therefore, the exploration of providing high-quality personalized service, carrying out interactive embodiment service and developing personalized interactive design technology of clothing will bring revolutionary influence to the clothing industry, which is also the next growth point and market height of clothing business.

\subsection{Broad economic prospects}

The year 2019 is called the first year of 5G, which marks the arrival of the era of 5G. It greatly reduces the delay of the AR/VR product system, increases the experience of the virtual environment, and ushered in a new take-off point of AR/VR technology. One area expected to have an impact on companies in the booming markets for AR and VR, and they are expected to play an increasingly important role in sectors such as real estate, retail, and manufacturing, according to technology market forecasters.

According to Panoramic Smart City Headquarters (2019), Gartner has reported that by 2020, 100 million consumers will shop online and in VR/AR stores. Goldman Sachs expects the AR/VR market to total $\$ 80$ billion by 2025 , roughly the size of the current PC market. Indeed, the company believes that the impact of AR and VR on the workplace and the way companies contact customers will be comparable to the transformation driven by smartphones. Statista, a German statistical database, is even more optimistic, predicting that the AR/VR industry will be worth $\$ 209$ billion by 2022 [5].

According to British PwC (2019)," Seeing is believing" looks at how virtual reality (VR) and augmented reality (AR) can add value to the economy and the benefits it could bring to manufacturing, healthcare, energy, retail, training, and development, which are expected to contribute a total of $\$ 1.5$ trillion to the global economy by 2030 [6]. The forecast and analysis of these well-known institutions in the industry are enough to show the strong market and broad economic prospects of AR and VR. Therefore, through the above discussion, it is feasible and necessary to develop and research the personalized clothing customization system based on AR technology.

\section{Overall design}

\subsection{System development model}

The development process of this system is based on one of the SDLC models, rapid 
application development (RAD). According to Whitten and Bentley (2008), developing applications with RAD requires six steps, as shown in Tablelbelow.

Table 1. Name of the table that RAD requires six steps [7].

\begin{tabular}{cc}
\hline Step & Content \\
\hline 1 & The analysis of problem and requirements \\
2 & Designing the application \\
3 & Developing the application \\
4 & Testing the application \\
5 & Refining the application \\
6 & Delivering the application \\
\hline
\end{tabular}

The steps of the system from step 2 to step 5 are an iterative process. After the developer passes the steps of designing, developing, testing, and perfecting the system, revise the design again, thus enter the process of re-development, each development, is to optimize the system, improve the quality of the process. The model is continuously improved to produce a satisfactory product.

\subsection{System specification}

The hardware and system environment used in this study for Based on AR Technology Personalized Cheongsam Customization System are as below.

Hardware. First, introduce the hardware used for developing the AR system in this study are: Smart Phone, Laptop, Camera, Mouse, Keyboard, Graphic Card, Marker (For triggering AR events), Azure Kinect. Software. Second, introduce the software environment for system development and testing, as shown in Table 2 below.

Table 2. Name of the table that the software environment for system development and testing.

\begin{tabular}{cc}
\hline Item & Tool \\
\hline Integrated Development & Visual Studio 2017 \\
Environment & Unity 2018.3.5 \\
Development Language & C\# \\
& Vuforia Augmented Reality SDK \\
& Azure Kinect DK SDK \\
Third Party Development & Android SDK \\
Libraries & OpenGL \\
& OpenNI 1.5.4.0 \\
& OpenCV 2.4.3 \\
Auxiliary Software & 3D Studio MAX 2012 \\
& Photoshop CS6 \\
\hline
\end{tabular}

System Requirements. Third, introduces the environment configuration of the system operation, as shown in Table 3 below.

\subsection{System functional architecture}

This system mainly implements the function of personalized clothing customization. Personalized clothing customization is mainly reflected in the three important links of the personalized design process, effect display to meet customer needs, and exquisite clothing production technology. The design process and the effect display link both involve the communication and collaboration between the merchant and the user. At the same time, 
hardware such as computers and Kinect cameras can be used as auxiliary equipment to better promote the design of clothing styles and colors, as well as the implementation of the effective display and try-on links. To this end, this system refers to the interactive function and technology-assisted links as intelligent interaction design modules, which are the key objects of this system. The two steps of the clothing design process and virtual try-in are merged into sub-modules of intelligent interaction modules. In the third link, the manual production process does not rely on science and technology and is independently set as a manual production module. Each module and sub-module have corresponding work content, and the system functional framework is shown in the Figure 1 below.

Table 3. Name of the table that the environment configuration of the system operation.

\begin{tabular}{ccc}
\hline NO. & Item & Parameter \\
\hline 1 & CPU & Intel(R) Core(TM) i7-4790 CPU \\
2 & GPU & @ 3.60GHz 4-cores \\
3 & RAM & Nvidia GeForce GTX 970 (4GB) \\
4 & OS & Windows 7 64-bit \\
5 & Somatosensory Equipment & Azure Kinect DK
\end{tabular}

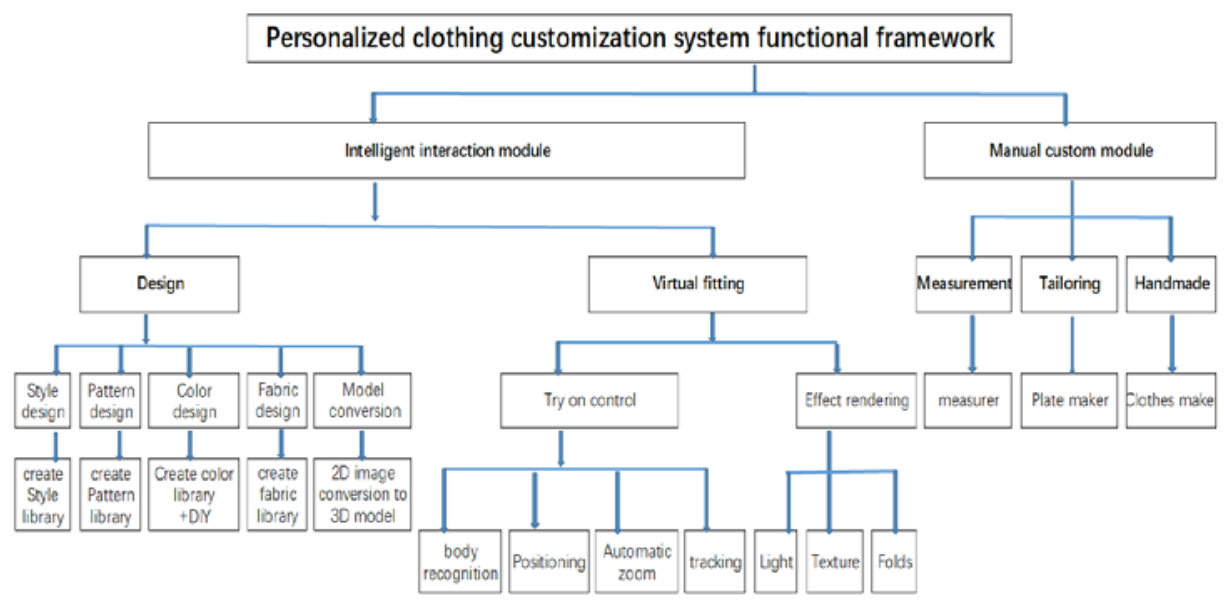

Fig. 1. The ystem FunctionFramework.

\subsection{System flow}

According to each functional module of the system, the system's workflow and the business logic of each module are now designed and constrained. The system working flow chart is shown in Fig. 2 below.

The design sub-module (as shown in Fig.2.A) is mainly for the detailed design of clothing style design, pattern design, color design, fabric design, etc., and also includes the conversion of $2 \mathrm{D}$ design samples to $3 \mathrm{D}$ clothing models. The system needs to build a corresponding material library for the selection of styles, patterns, colors, and fabrics for users to choose from. At the same time, in the color and pattern design, using the AR technology's coloring function can realize the user's independent color matching and self-creating pictures, achieving the effect of coloring and displaying immediately, allowing users to quickly observe the personal design effect. If the user is satisfied, then, determine the design draft and 
enter the trial-fitting step. Else, the user can return to the style design, pattern design, color design, and fabric design arbitrarily and redesign again until the user is satisfied and confirm the design draft. The design process can fully, quickly and conveniently complete the design process through the support of the material library, the guidance of the fashion designer and the customer's personal aesthetic choices, and determine the style of the custom clothing, which is conducive to increase customer participation and satisfaction, and also promoting design Improved Efficiency.

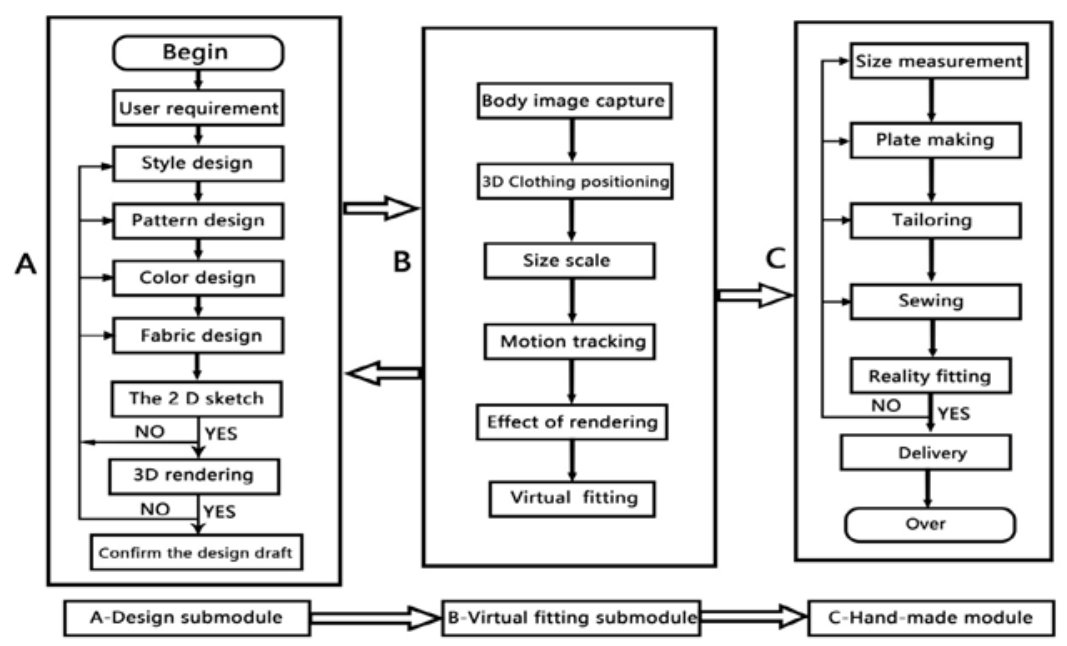

Fig. 2. The system working flow chart.

The virtual fitting sub-module (as shown in Fig.2.B) consists of two parts. The first part of this sub-module is the clothing control module, which is used to realize the function of accurate trying on virtual clothing. The specific process is to capture the depth data stream of human bones through the Azure Kinect DK somatosensory camera, and determine the image as "human" and human movement and position according to the recognition of the bone shape, so as to realize the bone tracking processing process, which is also to realize 3D clothing virtual try-on the proper position on the human body. At the same time, the system also hopes to apply the bone tracking method to set a scaling factor according to the corresponding proportion of bones caused by the distance from the camera after the human body moves. This factor is used to control the degree of scaling of the 3D clothing model to achieve the clothing model and human bone size-matching effect. The second part of this sub-module is effect rendering. Choose appropriate rendering tools to render clothing textures, lighting, dynamic collisions, and folds, etc., so that people can see virtual clothing more realistic effects through the display. Through virtual try-on, users can make customization decisions. If they are satisfied, they will enter the manual customization link. If they are not satisfied, they will return to the design sub-module and modify again until they are satisfied. The design sub-module and the virtual try-on sub-module together form an intelligent interaction module. This module enables users to participate in the design process and experience the effects of trial-wear, which can effectively promote users' satisfaction with designing clothing and improve the success rate of custom transactions.

The hand-made module (as shown in Fig.2.C) is implemented one by one from the four steps of a size measurement, garment plate making, fabric cutting, and fabric sewing according to the traditional garment manufacturing process. Each step needs to be hand-made by skilled professionals. The hand-made module focuses on the exquisite craftsmanship of custom clothing and the temperature of traditional hand-made, which is also the embodiment of custom clothing culture. 


\section{System evaluation}

Two technical aspects of the AR system developed in this study will be evaluated which are: the efficiency of the application and the effectiveness of the application in recognizing the $2 \mathrm{D}$ of a primitive object drawn.

Table 4. Name of the Table that verifiable variable effectiveness [8].

\begin{tabular}{cll}
\hline No. & \multicolumn{1}{c}{ Item } & \multicolumn{1}{c}{ Heuristic } \\
\hline 1 & $\begin{array}{l}\text { Do you know what is going on during all of the interactions? } \\
\text { If the camera or sensor detects more than one marker in the }\end{array}$ & Visibility of system status \\
2 & $\begin{array}{l}\text { User control and freedom } \\
\text { scene, is it possible to specify one? }\end{array}$ & User control and freedom \\
3 & $\begin{array}{l}\text { Is it possible to execute "role" or "undo" easily? (i.e., return to } \\
\text { a previous state without the virtual object) }\end{array}$ & Satisfaction \\
\hline
\end{tabular}

The instrument used in this study to evaluate the Augmented Reality application developed in this study is the checklist developed by Guimaraes \& Martins (2014). In Guimaraes \& Martins (2014) study, they had developed checklists to evaluate an Augmented Reality application by measuring its efficiency, effectiveness, and satisfaction [8]. However, only checklists that measure the application's effectiveness as shown in Table-IV and efficiency as shown in Table 4 are adopted to evaluate the usability of the AR application developed which is shown below.

Table 5. Name of the Table that verifiable variable efficiency [8].

\begin{tabular}{|c|c|c|}
\hline No. & Item & Heuristic \\
\hline 1 & $\begin{array}{l}\text { Is the loading time of virtual objects in the scene } \\
\text { satisfactory? }\end{array}$ & Visibility of system status \\
\hline 2 & $\begin{array}{l}\text { Are the virtual objects merged correctly with the real } \\
\text { world? (position, texture, scale) }\end{array}$ & $\begin{array}{l}\text { Match between system and the } \\
\text { real world }\end{array}$ \\
\hline 3 & $\begin{array}{l}\text { Is the virtual object animation coherent with the real } \\
\text { world? }\end{array}$ & $\begin{array}{l}\text { Match between system and the } \\
\text { real world }\end{array}$ \\
\hline 4 & $\begin{array}{l}\text { Are actions/feedback standardized? (e.g., borders are } \\
\text { added to the outside of the tracked object) }\end{array}$ & Consistency and standards \\
\hline 5 & $\begin{array}{l}\text { Is error prevention enabled? (i.e., if the user shows an } \\
\text { unexpected marker, is an error message presented to the } \\
\text { user?) }\end{array}$ & Error prevention \\
\hline 6 & $\begin{array}{l}\text { Is it easy to remember the application's functionalities? } \\
\text { (i.e., is it easy to memorize the functionalities of each } \\
\text { marker?) }\end{array}$ & Recognition rather than recall \\
\hline 7 & What is the learning curve like for novice users? & Flexibility and efficiency of use \\
\hline 8 & $\begin{array}{l}\text { Can expert users utilize the application in an optimized } \\
\text { manner? (e.g., can they skip introductory videos) }\end{array}$ & Flexibility and efficiency of use \\
\hline 9 & $\begin{array}{l}\text { Is it easy to stand the marker in an appropriate position } \\
\text { and orientation to be detected by the camera/sensor? }\end{array}$ & Flexibility and efficiency of use \\
\hline 10 & $\begin{array}{l}\text { Is the user instructed about what to do during the } \\
\text { interaction? (e.g., show the marker to the camera or is } \\
\text { there a manual) }\end{array}$ & $\begin{array}{l}\text { Help users recognise, diagnose, } \\
\text { and recover from errors }\end{array}$ \\
\hline 11 & $\begin{array}{l}\text { Are there specific requirements? (camera, marker, } \\
\text { mobile, GPS, user position, lighting, print, calibration) }\end{array}$ & Environment configuration \\
\hline 12 & Is the tracker system stable? & Accuracy \\
\hline 13 & $\begin{array}{l}\text { If the tracker system detects more than one object in the } \\
\text { scene, does the application continue to function correctly? }\end{array}$ & Accuracy \\
\hline
\end{tabular}


On the other hand, this study uses System Usability Scale (SUS) to test the comprehensive performance of the customized system. SUS is a standardized test questionnaire for product usability. It is a measurement of the overall perceived usability of the system. It was compiled by Brooke in 1986. According to the research of Tullis, T. S., \& Stetson, J. N., in the case of limited measurement data, the effectiveness of SUS system test is significantly higher than other similar test methods, that shown in Figure 3. [9] There are a total of 10 questions in the SUS questionnaire. The odd-numbered items are described positively, the odd-numbered items are scored as "original score -1 ", and the even-numbered items are described as negative. The scoring method is: "5-original score", the total is scored as a total of 10 questions*2.5 The coefficient is the SUS value. Finally, the results of the system evaluation are determined through the content in Figure 4.

$\%$ of "Correct" Conclusions

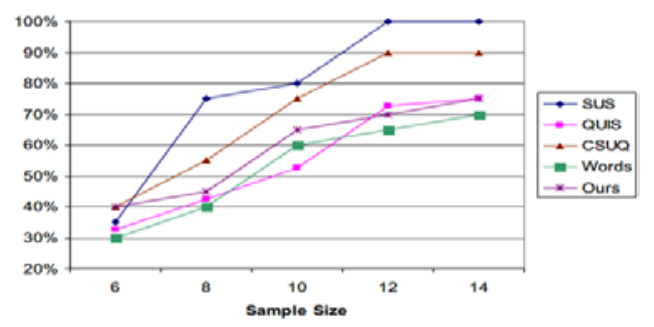

Fig. 3. SUS test efficiency[9].

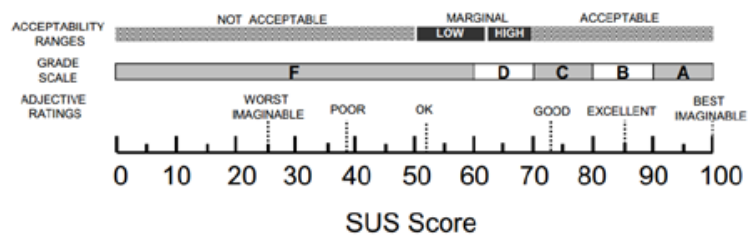

Fig. 4. SUS questionnaire content.

\section{Conclusion}

This paper analyzes the feasibility of the AR personalized clothing customization system, gives the overall system design scheme based on the RAD system development model, describes the workflow of each functional module in the system, and gives the system test method. This article shows a full-featured clothing customization system. This personalized clothing customization can be developed to provide a more interactive and interesting real time environment for clothing customization field. Since tailor often face problems in visualizing the objects in the personalized clothing customization design, especially to understand concepts which are abstract and not visible in our daily life, hence this real time interactive AR system tool has its own potential in aiding design processes of tailor because of its characteristic which allows user to visualise an animated object in 3D models based on their object choices. The design of this system solution can improve the satisfaction of clothing customization, reduce the production cost, and is conducive to the upgrade and development of the clothing customization industry. At the same time, the design of this system provides people with research references in the areas of AR system development, motion tracking, effect rendering, and virtual try-on. This new approach is to assist the users to resolve psychological difficulties interactively using AR technology besides the conventional exchange basic information with new people in the first personalized clothing customization design meeting approach. 
This study was supported by the 2019 Industry-University Cooperation Collaborative Education Project of the Ministry of Education of China ( project number: 201902015005), and the social science planning project of Hainan Province in 2020(project number: HNSK(YB)20-63).

\section{References}

1. Feng L , Ng G.W and Ma L 2020 A Review of An Interactive Augmented Reality Customization Clothing System Using Finger Tracking Techniques as Input Device. In: Alfred R., Lim Y., Haviluddin H., On C. (eds) Computational Science and Technology. Lecture Notes in Electrical Engineering, vol 603. (Singapore: Springer)

2. Michelle M 2012 Games Development with Unity, (China Beijing: Machinery Industry Press)

3. PAR Byron Consulting: 2018 Double Eleven retail record released. Retrieved from https://www.sohu.com/a/275144503_750243(2018).

4. China Business Industry Research Institute: Sales of "Double Eleven" in 2019 reached RMB 410.1 billion Yuan, ranking No. 1 in sales of personal care industry. Retrieved form http://finance.sina.com.cn/stock/ relnews/cn/ 2019-11-13/doc-iihnzahi0582606.shtml?from=wap(2019)

5. Panoramic Smart City Headquarters: The most popular and comprehensive interpretation of the important impact of 5G on VR / AR! Retrieved from https://www.sohu.com/a/315244770_120154841(2019)

6. PwC: Seeing is believing- How VR and AR will transform business and the economy. Retrieved from https://www.pwc.com/gx/en/industries/ technology/publications/economic-impact-of-vr-ar.html(2019).

7. Whitten J.L.and Bentley L.D. 2008 Introduction to system analysis and design. (New York: McGraw-HillInc)

8. Guimaraes, D.P., \& Martins, V.F. (2014). A checklist to evaluate augmented reality applications. In 2014 XVI Symposium on Virtual and Augmented Reality (SVR) (pp. 45-52). IEEE.

9. Tullis, T. S., \& Stetson, J. N. (2004).A compairson of questionnaires for assessing website usability. Proceedings of UPA 2004 conference. Minneapolis, Minnesota. 